\title{
Modification of two Parameters Rayleigh into three Parameters one Through Exponentiated
}

\author{
Waleed meaya rodeen \\ Basra university \\ Collage of management and economic \\ Statistics department
}

Recived : 25\10\2017

Revised : 9\1112017

Accepted : $19 \backslash 11 \backslash 2017$

Available online : 26/1/2018

DOI: 10.29304/jqcm.2018.10.1.356

\begin{abstract}
The continuous probability Rayleigh distribution is one of the important Is an important distribution that can be used To analyze signal data and statistical error data as well as time to failure, so we work on modifying two parameters Rayleigh $(\alpha, \theta)$ into three parameters one's $(\alpha, \theta, \lambda)$ through exponentiated, the new $p . d . f$ obtained, also the formula for $\mathrm{r}^{\text {th }}$ moments about origin is derived, to be used in estimating of parameters and also of reliability function. All derivation required were explained and the estimators by maximum likelihood and moments were obtained using different sets of initial values and the replicate of each experiment is $(R=1000)$, the results are compared by using mean squared error (MSE).
\end{abstract}

Keywords: Three Parameters Rayleigh, moments Estimators, (MOM), Maximum Likelihood Estimator (MLE), reliability Function $\left[R_{T}(t)\right]$, Mean Squared error (MSE).

\section{Introduction}

The Rayleigh distribution is one of the continuous probability distribution, which is one of the family of distribution introduced by, Burr (1942), it is used in analysis of signal and also in representing statistical errors of all types. Rayleigh distribution has many applications in representing statistical model for life time data (Lawless, J.F.[1982])[7], Dey, S. and das, M.K. (2007)[4], explain Bayesian approach interval for Rayleigh distribution. The p.d.f of two parameters Rayleigh $(\alpha, \theta)$ is given by;

$f(x ; \alpha, \theta)=$

$$
2 \alpha \theta^{2} x e^{-(\theta x)^{2}}\left(1-e^{-(\theta x)^{2}}\right)^{\alpha-1} \quad x, \alpha, \theta>0
$$

$$
0 \quad o / w
$$

Where $(\alpha)$ is the shape parameter and $(\theta)$ is the scale parameter.

The p.d.f in equation (1) may have different shape according to value of $(\alpha)$, if $\left(\alpha \leq \frac{1}{2}\right)$, the p.d.f in equation (1) is decreasing curve, and when $\left(\alpha>\frac{1}{2}\right)$, it is skewed to right.

The cumulative distribution function corresponding to equation (1) is;

$$
F(X ; \alpha, \theta)=\operatorname{pr}(X \leq x)=\left(1-e^{-(\theta x)^{2}}\right)^{\alpha}
$$

(2)

Also survival function is;

$S(X ; \alpha, \theta)=1-\left(1-e^{-(\theta x)^{2}}\right)^{\alpha}$

(3)

Also when $(\alpha \& \theta>0)$, we can define the hazard function;

$h(X ; \alpha, \theta)=\frac{f(X ; \alpha, \theta)}{S(X ; \alpha, \theta)}$

(4)

The p.d.f in equation (1) can be transformed to a three parameters Rayleigh through using exponentiated transformation by adding another shape parameter $(\lambda)$, using

$$
\begin{aligned}
& G_{X}(x)=\left[F(x, \alpha, \theta]^{\lambda}=\left[\left(1-e^{-(\theta x)^{2}}\right)^{\alpha}\right]^{\lambda}=\right. \\
& \left(1-e^{-(\theta x)^{2}}\right)^{\alpha \lambda} \\
& g(x)=\alpha \lambda\left(1-e^{-(\theta x)^{2}}\right)^{\alpha \lambda-1}\left(e^{-(\theta x)^{2}} 2 x \theta^{2}\right) \\
& =2 x \alpha \lambda \theta^{2} e^{-(\theta x)^{2}}\left(1-e^{-(\theta x)^{2}}\right)^{\alpha \lambda-1} \quad \alpha, \theta, \lambda> \\
& 0 \text { and } \alpha \lambda>1
\end{aligned}
$$

Equation (6) is the new p.d.f of exponentiated Rayleigh which have applications in signal and errors analysis. The survival function is: $S(X ; \alpha, \theta, \lambda)=1-\left(F(X ; \alpha, \theta)^{\lambda}\right.$ 


\section{Moment Estimator Method}

We find the formula for the $\mathrm{r}^{\text {th }}$ moments, about origin; $\mu_{r}^{\prime}=E\left(x^{r}\right)=\int_{0}^{\infty} x^{r} f(X ; \alpha, \theta)$

(7)

After some steps we have;

$\mu_{r}^{\prime}=\frac{\alpha}{\theta^{r}} \sum_{i=0}^{\alpha-1} \frac{C_{i}^{\alpha-1}(-1)^{\alpha-1-i}}{(\alpha-i)^{\frac{r}{2}+1}} \Gamma\left(\frac{r}{2}+1\right) \quad \alpha>1$

$\mu_{r}^{\prime}=E\left(x^{r}\right)=2 \alpha \lambda \theta^{2} \int_{0}^{\infty} x^{r+1} e^{-(\theta x)^{2}}(1-$

$e^{\left.-(\theta x)^{2}\right)^{\alpha \lambda-1}} d x$

Since

$$
(1-t)^{n}=\sum_{j=0}^{n} C_{j}^{n}(-1)^{j} t^{j}
$$

Then;

$$
\left(1-e^{-(\theta x)^{2}}\right)^{\alpha \lambda-1}=\sum_{j=0}^{\alpha \lambda-1} C_{j}^{\alpha \lambda-1}(-1)^{j}\left(e^{-(\theta x)^{2}}\right)^{j}
$$

$\mu_{r}^{\prime}$

$$
=2 \alpha \lambda \theta^{2} \sum_{j=0}^{\alpha \lambda-1} \int_{0}^{\infty} x^{r+1} e^{-(\theta x)^{2}} C_{j}^{\alpha \lambda-1}(-1)^{j}\left(e^{-j \theta^{2} x^{2}}\right) d x
$$

$\mu_{r}^{\prime}$

$=2 \alpha \lambda \theta^{2} \sum_{j=0}^{\alpha \lambda-1} C_{j}^{\alpha \lambda-1}(-1)^{j} \int_{0}^{\infty} x^{r+1} e^{-\theta^{2}(i+j) x^{2}} d x$

Let $\quad Z=\theta^{2}(1+j) x^{2} \quad \Rightarrow x=\frac{\sqrt{Z}}{\theta \sqrt{1+j}} \quad \Rightarrow$

$$
d x=\frac{1}{2 \theta \sqrt{Z} \sqrt{1+j}} d Z
$$

$\mu_{r}^{\prime}=k \int_{0}^{\infty}\left(\frac{\sqrt{Z}}{\theta \sqrt{1+j}}\right)^{r+1} e^{-Z} \frac{1}{2 \theta \sqrt{Z} \sqrt{1+j}} d Z$

$=k \frac{1}{2 \theta^{r+2}(\sqrt{1+j})^{r+1} \sqrt{1+j}} \int_{0}^{\infty} \sqrt{Z}^{r} e^{-Z} d Z$

$\mu_{r}^{\prime}=\frac{2 \alpha \lambda \theta^{2} \sum_{j=0}^{\alpha \lambda-1} C_{j}^{\alpha \lambda-1}(-1)^{j}}{2 \theta^{r+2}(\sqrt{1+j})^{r+2}} \int_{0}^{\infty} Z^{\frac{r}{2}} e^{-Z} d Z$

$=\alpha \lambda \sum_{j=0}^{\alpha \lambda-1} C_{j}^{\alpha \lambda-1}(-1)^{j} \frac{\Gamma\left(\frac{r}{2}+1\right)}{\theta^{r}}\left(\frac{1}{\sqrt{1+j}}\right)^{r+2} \quad \alpha \lambda$

$$
>1 \quad(11)
$$

By solving $\left(\mu_{r}^{\prime}=\frac{\sum_{i=1}^{n} x_{i}^{r}}{n}\right)$ for $(r=1,2,3)$ and $\alpha \lambda>$ 1 , we can obtain moments estimates for three parameters of (E-Ray).

\section{Maximum Likelihood Estimator}

Let $\left(x_{1}, x_{2}, \ldots, x_{n}\right)$ be a r. s. from p.d. $f, f(x ; \alpha, \theta)$, then;

$$
\begin{aligned}
& L=\prod_{i=1}^{n} f\left(x_{i}\right)=2^{n} \alpha^{n} \lambda^{n} \theta^{2 n} \prod_{i=1}^{n} x_{i} e^{-\theta^{2} \sum_{i=1}^{n} x_{i}^{2}} \prod_{i=1}^{n}(1 \\
& \left.-e^{-\theta^{2} x_{i}^{2}}\right)^{\alpha \lambda-1}
\end{aligned}
$$

$\log L=n \log 2+n \log \alpha+2 n \log \theta+n \log \lambda$

$$
\begin{aligned}
& +\sum_{i=1}^{n} \log x_{i}-\theta^{2} \sum_{i=1}^{n} x_{i}^{2} \\
& +(\alpha \lambda-1) \sum_{i=1}^{n} \log \left(1-e^{-\theta^{2} x_{i}^{2}}\right) \\
& \frac{\partial \log L}{\partial \alpha}=\frac{n}{\alpha}+\sum_{i=1}^{n} \log \left(1-e^{-\theta^{2} x_{i}^{2}}\right)=0 \\
& \hat{\alpha}_{M L E}=-\frac{n}{\sum_{i=1}^{n} \log \left(1-e^{-\theta^{2} x_{i}^{2}}\right)}
\end{aligned}
$$

$$
\begin{aligned}
& \frac{\partial \log L}{\partial \theta}=\frac{2 n}{\theta}-2 \theta \sum_{i=1}^{n} x_{i}^{2}+(\alpha \lambda \\
& \text {-1) } \sum_{i=1}^{n} \frac{(-1)\left(e^{-\theta^{2} x_{i}^{2}}\right)\left(-2 \theta x_{i}^{2}\right)}{\left(1-e^{-\theta^{2} x_{i}^{2}}\right)} \\
& =0 \\
& (\alpha \lambda-1) \sum_{i=1}^{n} \frac{(-1)\left(e^{-\theta^{2} x_{i}^{2}}\right)\left(-2 \hat{\theta} x_{i}^{2}\right)}{\left(1-e^{-\theta^{2} x_{i}^{2}}\right)}=\hat{\theta} \sum_{i=1}^{n} x_{i}^{2}-\frac{n}{\hat{\theta}} \\
& \hat{\theta}^{2}\left[\sum_{i=1}^{n} x_{i}^{2}-(\alpha \lambda-1) \sum_{i=1}^{n} \frac{(-1)\left(e^{-\theta^{2} x_{i}^{2}}\right)\left(-2 \theta x_{i}^{2}\right)}{\left(1-e^{-\theta^{2} x_{i}^{2}}\right)}\right] \\
& =n
\end{aligned}
$$

By using newton raphson method to get the parameter estimator of $\theta$

\section{Simulation}

The estimation and comparison has been done through simulation procedures, were the values are generated from;

$$
\begin{gathered}
G_{X}(x)=\left[\left(1-e^{-(\theta x)^{2}}\right)^{\alpha}\right]^{\lambda} \\
u_{i}=\left[\left(1-e^{-\theta^{2} x^{2}}\right)\right]^{\alpha \lambda} \\
u_{i}^{\frac{1}{\alpha \lambda}}=\left(1-e^{-\theta^{2} x^{2}}\right) \\
e^{-\theta^{2} x^{2}}=1-u_{i}^{\frac{1}{\alpha \lambda}} \\
-\theta^{2} x^{2}=\ln \left(1-u_{i}^{\frac{1}{\alpha \lambda}}\right) \\
x^{2}=-\frac{1}{\theta^{2}} \ln \left(1-u_{i}^{\frac{1}{\alpha \lambda}}\right) \\
x_{i}=-\frac{1}{\theta} \sqrt{\ln \left(1-u_{i}^{\frac{1}{\alpha \lambda}}\right)}
\end{gathered}
$$


The initial values are;

\begin{tabular}{|c|c|c|}
\hline$\alpha$ & $\lambda$ & $\theta$ \\
\hline 3 & 0.5 & 0.8 \\
\hline 4 & 0.8 & 1.5 \\
\hline 3.5 & 2 & 2 \\
\hline
\end{tabular}

Table (1): Estimation of $(\alpha=3, \theta=0.6, \lambda=0.5)$

\begin{tabular}{|c|c|c|c|c|c|c|}
\hline$n$ & $\hat{\theta}_{M L E}$ & $\hat{\theta}_{M O M}$ & $\hat{\lambda}_{M L E}$ & $\hat{\lambda}_{M O M}$ & $\hat{\alpha}_{M L E}$ & $\hat{\alpha}_{M O M}$ \\
\hline \multirow{10}{*}{$\begin{array}{l}3 \\
0\end{array}$} & 0.6604 & $\begin{array}{c}0.5159 \\
3\end{array}$ & 0.5506 & 0.5560 & 2.4756 & 2.8660 \\
\hline & 0.6403 & $\begin{array}{c}0.5227 \\
4\end{array}$ & 0.5643 & 0.5641 & 2.4814 & 2.9520 \\
\hline & 0.5031 & $\begin{array}{c}0.5149 \\
9\end{array}$ & 0.5602 & 0.6203 & 2.4703 & 2.9830 \\
\hline & 0.8703 & $\begin{array}{c}1.0406 \\
1\end{array}$ & 0.8613 & 0.6614 & 2.9611 & 2.8820 \\
\hline & 0.8832 & 1.4035 & 0.9921 & 1.0021 & 3.0112 & 1.3846 \\
\hline & 0.9994 & 1.0362 & 1.3011 & 1.0314 & 3.0611 & 1.4157 \\
\hline & 1.4683 & 1.5266 & 1.2920 & 1.1121 & 3.5211 & 1.3992 \\
\hline & 1.5962 & 1.9821 & 1.0122 & 1.2306 & 3.5311 & 1.3672 \\
\hline & 1.6401 & 1.9932 & 1.0089 & 1.5216 & 3.8020 & 1.4407 \\
\hline & 1.6612 & 1.9961 & 1.5283 & 1.5220 & 3.8840 & 1.4402 \\
\hline \multirow{10}{*}{$\begin{array}{l}6 \\
0\end{array}$} & 0.48776 & 0.5006 & 1.4407 & 1.4837 & 1.1254 & 1.5267 \\
\hline & 0.49582 & 0.5832 & 1.4408 & 1.4962 & 1.3621 & 1.5382 \\
\hline & 0.51084 & 0.9486 & 1.4425 & 0.9437 & 1.6410 & 1.5171 \\
\hline & 0.8736 & 0.5044 & 0.8963 & 0.9882 & 1.0407 & 1.5189 \\
\hline & 0.9921 & 0.9936 & 0.8902 & 0.9945 & 1.0475 & 1.2346 \\
\hline & 0.9914 & 1.0052 & 0.8831 & 1.6931 & 1.5743 & 1.2204 \\
\hline & 1.4001 & 1.0069 & 0.8801 & 1.4825 & 1.5675 & 1.0666 \\
\hline & 1.4231 & 1.5022 & 0.8711 & 1.5119 & 1.0161 & 1.2431 \\
\hline & 1.4303 & 1.5311 & 0.6531 & 1.4945 & 1.4241 & 1.3352 \\
\hline & 1.4996 & 1.4958 & 0.6420 & 1.8462 & 1.2563 & 1.2453 \\
\hline \multirow{10}{*}{$\begin{array}{l}9 \\
0\end{array}$} & 0.49784 & 0.5042 & 0.5046 & 0.4871 & 2.1106 & 1.4837 \\
\hline & 0.49987 & 0.4991 & 0.5049 & 0.4973 & 2.004 & 1.4810 \\
\hline & 0.49417 & 1.0081 & 0.4998 & 0.4917 & 2.001 & 1.4707 \\
\hline & 0.9981 & 1.0005 & 0.4999 & 0.9855 & 1.889 & 0.9463 \\
\hline & 0.99046 & 1.0411 & 1.0005 & 0.9965 & 1.992 & 1.6472 \\
\hline & 1.00231 & 1.5432 & 1.0024 & 0.9943 & 1.0115 & 1.5321 \\
\hline & 1.51951 & 1.5171 & 1.0114 & 0.9931 & 1.5341 & $\begin{array}{c}1.5522 \\
1\end{array}$ \\
\hline & $\begin{array}{c}1.50100 \\
9\end{array}$ & $\begin{array}{c}1.5189 \\
1\end{array}$ & 1.5348 & 1.5171 & $\begin{array}{c}1.5171 \\
8\end{array}$ & 1.4841 \\
\hline & 1.50372 & 1.3264 & $\begin{array}{c}1.5171 \\
6\end{array}$ & $\begin{array}{c}1.5188 \\
6\end{array}$ & $\begin{array}{c}1.5170 \\
6\end{array}$ & 1.4742 \\
\hline & 1.64021 & 1.4302 & 1.5189 & 1.5002 & 1.5051 & 1.3762 \\
\hline
\end{tabular}

Table (2): Estimators of Reliability Function.

\begin{tabular}{|c|c|c|c|c|c|c|}
\hline$n$ & $\alpha$ & $\lambda$ & $\theta$ & $\hat{R}_{M L E}$ & $\hat{R}_{M O M}$ & Best \\
\hline \multirow{9}{*}{30} & \multirow{3}{*}{3} & 0.5 & 0.8 & 0.68323 & 0.59871 & MLE \\
\hline & & 0.8 & 1.5 & 0.76112 & 0.66082 & MLE \\
\hline & & 0.6 & 2 & 0.79014 & 0.89314 & MOM \\
\hline & \multirow{3}{*}{4} & 0.5 & 0.8 & 0.99061 & 0.88541 & MLE \\
\hline & & 0.8 & 1.5 & 0.9843 & 0.93061 & MLE \\
\hline & & 0.6 & 2 & 0.60075 & 0.77431 & MOM \\
\hline & \multirow{3}{*}{3.5} & 0.5 & 0.8 & 0.99941 & 0.98405 & MLE \\
\hline & & 0.8 & 1.5 & 0.78063 & 0.69904 & MLE \\
\hline & & 0.6 & 2 & 0.66421 & 0.63415 & MLE \\
\hline \multirow{9}{*}{60} & \multirow{3}{*}{3} & 0.5 & 0.8 & 0.77435 & 0.90763 & MOM \\
\hline & & 0.8 & 1.5 & 0.84306 & 0.77531 & MOM \\
\hline & & 0.6 & 2 & 0.872310 & 0.99078 & MLE \\
\hline & \multirow{3}{*}{4} & 0.5 & 0.8 & 0.843301 & 0.884693 & MOM \\
\hline & & 0.8 & 1.5 & 0.706492 & 0.77084 & MLE \\
\hline & & 0.6 & 2 & 0.77145 & 0.84773 & MLE \\
\hline & \multirow{3}{*}{3.5} & 0.5 & 0.8 & 0.78715 & 0.77806 & MLE \\
\hline & & 0.8 & 1.5 & 0.84734 & 0.84707 & MOM \\
\hline & & 0.6 & 2 & 0.85321 & 0.9993 & MOM \\
\hline \multirow{9}{*}{90} & \multirow{3}{*}{3} & 0.5 & 0.8 & 0.99061 & 0.99341 & MOM \\
\hline & & 0.8 & 1.5 & 0.84667 & 0.84732 & MOM \\
\hline & & 0.6 & 2 & 0.83552 & 0.84352 & MOM \\
\hline & \multirow{3}{*}{4} & 0.5 & 0.8 & 0.847076 & 0.84352 & MLE \\
\hline & & 0.8 & 1.5 & 0.776171 & 0.77061 & MLE \\
\hline & & 0.6 & 2 & 0.89031 & 0.84315 & MLE \\
\hline & \multirow{3}{*}{3.5} & 0.5 & 0.8 & 0.88061 & 0.9091 & MOM \\
\hline & & 0.8 & 1.5 & 0.84553 & 0.9312 & MOM \\
\hline & & 0.6 & 2 & 0.84321 & 0.9906 & MOM \\
\hline
\end{tabular}


Table (3): Mean square error for $\hat{R}$

\begin{tabular}{|c|c|c|c|c|c|c|}
\hline$n$ & $\alpha$ & $\lambda$ & $\theta$ & $\hat{R}_{M L E}$ & $\hat{R}_{M O M}$ & Best \\
\hline \multirow{9}{*}{30} & \multirow{3}{*}{3} & 0.5 & 0.8 & 0.0041809 & 0.002567 & ML \\
\hline & & 0.8 & 1.5 & 0.0026424 & 0.0031071 & $\mathrm{ML}$ \\
\hline & & 0.6 & 2 & 0.002747 & 0.0033751 & MOM \\
\hline & \multirow{3}{*}{4} & 0.5 & 0.8 & 0.003617 & 0.00214 & ML \\
\hline & & 0.8 & 1.5 & 0.004122 & 0.00431 & MOM \\
\hline & & 0.6 & 2 & 0.001513 & 0.00221 & MOM \\
\hline & \multirow{3}{*}{3.5} & 0.5 & 0.8 & 0.001643 & 0.004151 & MOM \\
\hline & & 0.8 & 1.5 & 0.003512 & 0.00421 & MOM \\
\hline & & 0.6 & 2 & 0.003415 & 0.00396 & MOM \\
\hline \multirow{9}{*}{60} & \multirow{3}{*}{3} & 0.5 & 0.8 & 0.000436 & 0.000412 & ML \\
\hline & & 0.8 & 1.5 & 0.000434 & 0.000251 & ML \\
\hline & & 0.6 & 2 & 0.000104 & 0.000161 & ML \\
\hline & \multirow{3}{*}{4} & 0.5 & 0.8 & 0.000974 & 0.000994 & MOM \\
\hline & & 0.8 & 1.5 & 0.000762 & 0.000636 & ML \\
\hline & & 0.6 & 2 & 0.000152 & 0.000051 & ML \\
\hline & \multirow{3}{*}{3.5} & 0.5 & 0.8 & 0.000734 & 0.000626 & ML \\
\hline & & 0.8 & 1.5 & 0.000346 & 0.000215 & ML \\
\hline & & 0.6 & 2 & 0.000789 & 0.000313 & ML \\
\hline \multirow{9}{*}{90} & \multirow{3}{*}{3} & 0.5 & 0.8 & 0.000919 & 0.0001021 & ML \\
\hline & & 0.8 & 1.5 & 0.000514 & 0.000231 & ML \\
\hline & & 0.6 & 2 & 0.000501 & 0.000282 & ML \\
\hline & \multirow{3}{*}{4} & 0.5 & 0.8 & 0.000747 & 0.0002031 & ML \\
\hline & & 0.8 & 1.5 & 0.000671 & 0.000115 & ML \\
\hline & & 0.6 & 2 & 0.000621 & 0.000071 & ML \\
\hline & \multirow{3}{*}{3.5} & 0.5 & 0.8 & 0.000606 & 0.0000241 & ML \\
\hline & & 0.8 & 1.5 & 0.000264 & 0.0000112 & ML \\
\hline & & 0.6 & 2 & 0.000255 & 0.0000151 & ML \\
\hline
\end{tabular}

\section{Conclusion}

凶 Rayleigh distribution is a good probability distribution for representing signal data, also time to failure for medical physical experiments when failure happens after $(t>\alpha$ for example), but in our research we work on modifying two parameters Rayleigh into three one's.

囚eliability estimators by moments and maximum likelihood indicates that $\left(\widehat{R}_{M L E}\right)$ is better than $\left(\hat{R}_{M O M}\right)$ with percentage $(75 \%)$, while $\left(\hat{R}_{\text {MOM }}\right)$ with $(25 \%)$.

冈 The sample size taken are $(n=30,60,90)$, with different sets of initial values obtained under condition $(\alpha \lambda>1)$.

\. The results are compared by statistical measures mean squares errors.

\section{References}

[1]Abd - Elfatta, A.M., (2011), "Goodness of fit test for the generalized Rayleigh distribution with unknown parameters", Journal of Statistical Computation and Simulation, Vol.81, No.3, 357 - 366 .

[2]Abdul-Moniem I. B. and Abdul-Hameed H.F. (2012),“On Exponentiated Lomax Distribution”.Inter. Jou. Of Math. Archive -3(5), pp:2144-2150.

[3]Azeem Ali, Syed AnwerHasnain, and Munir Ahmad, (2015), MODIFIED BURR III DISTRIBUTION, PROPERTIES AND APPLICATIONS, Pak. J. Statist.Vol. 31(6), 697-708.

[4]Dey, S. and Das, M.K. (2007), “A note on Prediction Interval for a Rayleigh Distribution: Bayesian Approach", American Journal of Mathematical and Management Science, 27(1\&20, 43 - 48.

[5]Flaih A., Elsallaukh H., Mandi E. and Milanova M. (2012), "The Exponentiated Inverted WeibullDistribution", Appl. Math. Inf. Sci., vol. 6, no. 2, pp:167-171.

[6]Kundu, D. and Raqab, M.Z., (2005), "Generalized Rayleigh Distribution: Different Methods of Estimations", Computational Statistical and Data Analysis, 49, 187 - 200.

[7]Lawless, J.F., (1982), "Statistical Models and Methods for life time data", Jhon Wiley \& Sons, New York.

[8]MOHAMMAD Z. RAQAB - MOHAMED T. MADI, (2009), "Bayesian analysis for the exponentiated Rayleigh distribution", METRON International Journal of Statistics 2009, vol. LXVII, n. 3, pp. 269-288.

[9]Raqab, M.Z., (2005), “Generalized Rayleigh Distribution Different Methods of Estimation", Computational Statistics and Data Analysis, 49,187 200.

[10]Raqab M.Z. and Madi M.T. (2009), "Bayesian Analysis for the Exponentiated Rayleigh Distribution", METRON-Inter. J. of stal., vol. 67, no.3, pp:269-288.

[11]Rosaiaah, K., Kantam, R.R.L. and Santosh Kumar, Ch., (2007), Reliability of Test plants for exponentiated log logistic distribution", Economic Quality Control, Vol. , 165 - 175.

[12]Reza Azimi1, FarhadYaghmaei and DarioshAzim, (2012)," Comparison of Bayesian Estimation Methods for Rayleigh Progressive Censored Data Under the Different Asymmetric Loss Function", International Journal of Applied Mathematical Research, 1 (4) (2012) 452-461.

[13]SankuDey, TanujitDey, Sudhansu S. Maiti, (2015), "Bayes Shrinkage Estimation of the Parameter of Rayleigh Distribution for Progressive Type-II Censored Data", Austrian Journal of Statistics, Volume 44, 3-15. 
[14]Sanko Dey (2008), "Minimax Estimation of the parameter of the Rayleigh distribution under quadratic loss function", Data Science Journal, Vol. 7, 23.

[15]Shankar Kumar Shrestha, Vijay Kumar, (2014), "Bayesian Analysis for the Generalized RayleighDistribution", International Journal of Statistika and Mathematika, Volume 9, Issue 3, pp 118131.
[16]Smail Mahdi, (2006), "Improved Parameter Estimation in RayleighModel", Metodološkizvezki, Vol. 3, No. 1, 63-74.

وليد مية رودين

جامعة البصرة / كلية الادارة والاقتصاد / قودم الاحصاء

يعتبر توزيع رايلي من التوزيعات المستمرة المهمه التي تستخدم في بيانات اوقات الفتل ، اذ تم عمل تحديث على توزيع

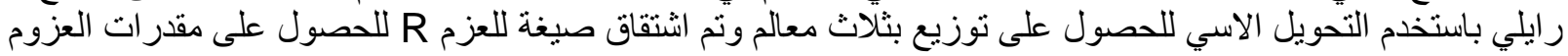

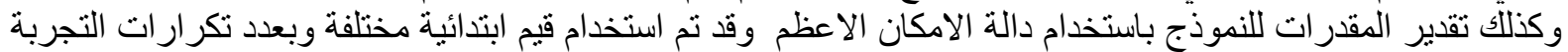
لـ(R=1000) وتم مقارنه النتائج بالاعتماد على معيار منوسط مربعات الخطاء( 